\title{
Le double visage de la réserve naturelle des Terres australes
} françaises

Mita Manouvel, Eric Naim-Gesbert

\section{Citer ce document / Cite this document :}

Manouvel Mita, Naim-Gesbert Eric. Le double visage de la réserve naturelle des Terres australes françaises. In: Revue Juridique de l'Environnement, $n^{\circ} 4,2007$. pp. 445-455;

doi : https://doi.org/10.3406/rjenv.2007.4673

https://www.persee.fr/doc/rjenv_0397-0299_2007_num_32_4_4673

Fichier pdf généré le 04/04/2018 


\title{
Résumé
}

Un décret du 3 octobre 2006 fait des Terres australes françaises une réserve naturelle. Ces Terres se composent de plusieurs groupes d'îles (deux archipels : Kerguelen et Crozet, deux îles : Saint-Paul et Amsterdam). Une partie du continent antarctique - la Terre Adélie - non visée par le décret, forme avec ces îles les Terres australes et antarctiques françaises. La réserve naturelle est l'instrument privilégié de la préservation du patrimoine écologique. Elle permet l'établissement d'un nouvel ordre juridique conciliant les exigences de la préservation de la biodiversité tout en maintenant les potentialités d'exploitation des ressources halieutiques. En effet, si la réserve naturelle des Terres australes est bien une réserve nationale terrestre, elle n'est pas une réserve maritime.

\begin{abstract}
A decree issued on October 3, 2006 has turned the French Austral Territories into a wildlife reserve. The French Austral Territories are made up of several groups of islands - two archipelagoes, Kerguelen and Crozet, and two islands, St Paul and Amsterdam. Adelie Land, in Antarctica, is not concerned by the decree ; with these islands it makes up the French Austral and Antarctic Territories. Wildlife reserves are the most favoured instruments of preservation of the environment. They make it possible to establish a new legal order which reconciles the necessity of biodiversity preservation with the continuation of the exploitation of fishing resources : the French Austral Territories wildlife reserve is indeed a land reserve, not a marine reserve.
\end{abstract}




\section{LE DOUBLE VISAGE DE LA RÉSERVE NATURELLE DES TERRES AUSTRALES FRANÇAISES}

Mita MANOUVEL

Maître de conférences HDR à l'Université de la Réunion

et Eric NAIM-GESBERT

Maître de conférences HDR à l'Université de la Réunion

Expert en droit de l'environnement a I'UNITAR

(United Nations Institute for Training and Research)

Résumé Un décret du 3 octobre 2006 fait des Terres australes françaises une réserve naturelle. Ces Terres se composent de plusieurs groupes d'îles (deux archipels : Kerguelen et Crozet, deux îles : Saint-Paul et Amsterdam). Une partie du continent antarctique - la Terre Adélie - non visée par le décret, forme avec ces îles les Terres australes et antarctiques françaises. La réserve naturelle est l'instrument privilégié de la préservation du patrimoine écologique. Elle permet l'établissement d'un nouvel ordre juridique conciliant les exigences de la préservation de la biodiversité tout en maintenant les potentialités d'exploitation des ressources halieutiques. En effet, si la réserve naturelle des Terres australes est bien une réserve nationale terrestre, elle n'est pas une réserve maritime.

Summary A decree issued on October 3, 2006 has turned the French Austral Territories into a wildlife reserve. The French Austral Territories are made up of several groups of islands - two archipelagoes, Kerguelen and Crozet, and two islands, St Paul and Amsterdam. Adelie Land, in Antarctica, is not concerned by the decree; with these islands it makes up the French Austral and Antarctic Territories. Wildlife reserves are the most favoured instruments of preservation of the environment. They make it possible to establish a new legal order which reconciles the necessity of biodiversity preservation with the continuation of the exploitation of fishing resources: the French Austral Territories wildlife reserve is indeed a land reserve, not a marine reserve. 
Un décret du 3 octobre $2006^{1}$ porte création de la réserve naturelle des Terres australes françaises. Celles-ci se composent de trois groupes dîles situées dans la partie sud de l'océan Indien. Le premier groupe d'îles est formé par l'archipel des Crozet, le second par l'archipel des Kerguelen, le troisieme par les îles Saint-Paul et Amsterdam. Les deux premiers se trouvent dans la zone de convergence antarctique ${ }^{2}$, le troisieme beaucoup plus au Nord. Ces trois groupes d'îles forment avec la Terre Adélie, morceau du continent antarctique, le Territoire des Terres australes et antarctiques françaises (TAAF). II est à souligner que ces quatre composantes des TAAF sont séparées les unes des autres par des milliers de kilometres ${ }^{3}$. Il est à noter également que la Terre Adélie n'est pas visée par le décret, puisqu'elle est déjà partie intégrante de la " réserve naturelle, consacrée à la paix et à la science " instituée par l'article 2 du Protocole de Madrid du 4 octobre 1991.

Une brève présentation législative et administrative des TAAF contribuera à l'intelligence des problèmes que nous nous proposons d'évoquer ${ }^{4}$. Les prises de possession de ces territoires se font en 1772 (Kerguelen et Crozet), en 1840 (Terre Adélie) et 1843 (Saint-Paul et Amsterdam). Longtemps oubliés des gouvernements français, les différents territoires sont rattachés administrativement au Gouvernement général de Madagascar par un décret du 21 novembre 1924 qui scelle ainsi leur unite de destin. Les TAAF sont ensuite érigées en territoire d'outre-mer par une loi du 6 août 1955 et dotées de l'autonomie administrative et financière. Le siège du territoire d'abord établi à Paris est transféré à la Réunion en 1996. Il s'agissait, il est vrai, d'un territoire d'outre-mer bien singulier puisqu'il était dépourvu de population.

Depuis la réforme constitutionnelle du 28 mars 2003, la loi détermine le régime législatif et l'organisation particulière des Terres australes et antarctiques françaises ${ }^{5}$. Un projet de loi, portant dispositions statutaires et institutionnelles relatives à l'outre-mer, déposé sur le bureau du Sénat en mai 2006, est actuellement en cours d'examen ${ }^{6}$. Les dispositions saillantes concernant le statut des TAAF portent sur le rattachement des îles Eparses de l'océan Indien ${ }^{7}$ au territoire des TAAF, la simplification de son régime législatif et la modification de la composition et du fonctionnement du conseil consultatif chargé d'assister l'Administrateur supérieur. II s'agit donc davantage d'un toilettage que d'une réforme de fond.

Le territoire est dirigé par un Administrateur supérieur. Nommé par décret, il est le dépositaire des pouvoirs de la République dans le territoire et reçoit ses instructions par l'intermediaire du ministre de l'Outre-Mer. II promulgue les lois et décrets applicables au territoire ; il est chargé de leur exécution ainsi que de

1. JO du 4 octobre.

2. La zone antarctique. telle que définie par le Traité de Washington du $1^{\text {er }}$ décembre 1959 , est la région située au sud du $60^{\circ}$ degré de latitude Sud. L'archipel des Crozet est situé par $46^{\circ}$ à $46^{\circ} 30^{\prime}$ de latitude Sud, l'archipel des Kerguelen par $49^{\circ}$ de latitude Sud. Les îles Saint-Paul et Amsterdam sont situées respectivement par $37^{\circ} 50^{\prime}$ et $38^{\circ} 42^{\prime}$ de latitude Sud.

3. Plus de $4000 \mathrm{~km}$ entre la Terre Adélie et l'archipel des Crozet, plus de $1400 \mathrm{~km}$ entre l'archipel des Crozet et l'archipel des Kerguelen, plus de $1400 \mathrm{~km}$ également entre l'archipel des Crozet et Saint-Paul et Amsterdam.

4. Pour une présentation plus complète, voir M. Manouvel, "Le territoire d'outre-mer des Terres australes et antarctiques françaises, aspects de droit interne, aspects de droit international ", préface de J.- P. Charpentier, Paris, Montchrestien, 2000, 219 pages.

5. Nouvel article 72-3 de la Constitution.

6. Le rapporteur de la Commission des lois du Sénat, C. Cointat, a déposé son rapport le 18 octobre 2006.

7. Juan de Nova, Bassas da India, Europa, Les Glorieuses, dans le canal du Mozambique et Tromelin au Nord de la Réunion. Sans être rattachées organiquement aux TAAF, ces îles étaient déjà, depuis 2005, gérées par l'Administrateur supérieur des TAAF. 
l'exécution des décisions du Gouvernement. II dirige l'action des services de l'Etat ${ }^{8}$. Le territoire a été divisé, en 1956, en quatre circonscriptions correspondant aux unités géographiques : district de Saint-Paul et Amsterdam, district de Kerguelen, district de Crozet et district de Terre Adélie. Ces circonscriptions administratives sont dirigées par un chef de district, représentant permanent de l'Administrateur supérieur. C'est donc tout naturellement que l'Administrateur supérieur est chargé par le décret du 3 octobre 2006 « de la gestion de la réserve naturelle » dans les districts austraux.

L'Administrateur supérieur est assisté dans sa tâche par deux organes consultatifs. Le premier est le Conseil consultatif, composé actuellement de sept membres nommés par le ministre de l'Outre-Mer pour cinq ans ${ }^{9}$. Le Conseil consultatif tient lieu de comité consultatif de la nouvelle réserve naturelle. Le second est le Comité de l'environnement, créé en 1982, et consulté par l'Administrateur supérieur sur les questions de protection de la nature, de sauvegarde des sites, de lutte contre les nuisances. Le territoire n'ayant plus de conseil scientifique ${ }^{10}$, c'est le Comité de l'environnement polaire institué par le décret du 29 mars 1993 (modifié le 9 avril 2002) qui va tenir lieu de conseil scientifique de la réserve naturelle ${ }^{11}$. Soulignons que ce comité, institué auprès du ministre de l'Environnement, n'est pas un organe consultatif des TAAF même s'il peut être saisi par l'Administrateur supérieur.

La création de cette réserve naturelle nationale est un élément majeur de la Stratégie nationale de la biodiversité mise en œuvre par le ministère de l'Ecologie et du Développement durable. Ce choix peut surprendre. L'on eût pu imaginer la création d'un parc national moderne, d'une part, en raison de la préexistence d'un pseudo parc national issu d'un décret de 1938, d'autre part, du fait de la refonte législative (loi du 14 avril 2006) du statut général du parc national intégrant l'objectif de développement durable et créant un dérivé : le parc naturel marin $^{12}$. II en est autrement. Les causes de ce choix tiennent d'abord à la fonction première de la réserve naturelle; c'est l'instrument privilégié de la préservation du patrimoine écologique, adapté et évolutif, fondé au mieux sur les exigences scientifiques, en particulier comme cadre de la recherche. Et puis, de facto, s'établit une correspondance sémantique avec le statut international de la Terre Adélie en vertu du Protocole de Madrid précité. Enfin, et surtout, les

8. Notons toutefois que c'est le préfet de la Réunion qui dirige la zone de défense dont relèvent les TAAF et que c'est encore le préfet de la Réunion qui est délégué du Gouvernement dans les eaux bordant les côtes des terres françaises de l'océan Indien et des Terres australes et antarctiques françaises.

9. Cinq d'entre eux sont nommés au titre de différents ministères (Défense, Outre-Mer, Education nationale et recherche, Transports, Mer), les deux derniers sont désignés par le ministre de l'Outre-Mer parmi les personnalites ayant participé à des missions scientifiques dans les îles australes et antarctiques françaises. Le projet de loi actuellement en examen devrait elargir sa composition.

10. Un conseil scientifique avait été crée en 1965. Il comprenait dix-sept membres nommés par l'Administrateur supérieur et chargés de l'assister dans l'étude des questions scientifiques et dans la définition des programmes de recherche. II n'existe plus depuis 1992, la mission recherche du territoire ayant été transférée des TAAF à l'Institut français pour la recherche et la technologie polaire (actuel Institut polaire). 11. Les dix membres du comité autres que le président sont nommés dans les conditions suivantes: deux sur proposition du ministre chargé des affaires étrangères, deux sur proposition du ministre chargé de l'environnement, deux sur proposition du ministre chargé des départements et territoires d'outre-mer. deux sur proposition du ministre chargé de la recherche et deux sur proposition du Conseil national de la protection de la nature.

12. E. Naim-Gesbert, "Le nouveau droit du parc national entre fidélité généalogique el développement durable », Recueil Dalloz, $n^{\circ} 30,7$ septembre 2006, p. 2061-2065. S. Mabile, "Les parcs naturels marins consacrés par le législateur », RJE, $n^{\circ} 3 / 2006$, p. 251-264. 
dispositions législatives du Code de l'environnement, définissant le régime juridique du parc national, ne sont pas applicables à ces territoires ${ }^{13}$.

C'est un acte à double visée : consolider la préservation de l'environnement de ces territoires extrêmes, îles océaniques du sud de l'océan Indien au patrimoine écologique riche et au potentiel de recherche avéré sur la biodiversité ou l'évolution du climat, tout en préservant les intérêts économiques actuels et futurs dans les eaux des Terres australes françaises.

\section{I. - LA CONSOLIDATION}

\section{DE LA PROTECTION DE L'ENVIRONNEMENT DANS LES TERRES AUSTRALES FRANÇAISES}

La création d'une réserve naturelle dans ces groupes d'îles se justifie doublement : d'un point de vue scientifique pour préserver le patrimoine biologique, sanctuaire et observatoire privilégié, en droit comme solution à la nécessaire rationalisation du droit existant. La création de la réserve naturelle met un terme à ce maquis, clarifie la situation juridique en instaurant un nouvel ordre juridique.

\section{A) A CONFIGURATION NATURELLE EXTRÊME,} DROIT COMPLEXE?

Les Terres australes françaises, éloignées des sphères de l'activité humaine, sans population permanente, disposent d'un patrimoine biologique d'exception, notamment du fait de stratégies adaptatives remarquables des espèces et des écosystèmes au cours de l'évolution. II en est d'autant plus vulnérable. Les lois écologiques disent la fragilité des especes et des écosystèmes sur de petits territoires, en particulier insulaires; précisément, la science enseigne que les communautés insulaires présentent, à conditions écologiques equivalentes, des particularités distinctes des communautés continentales (population réduite, moindre fécondité, etc.) ${ }^{14}$. Les Terres australes en sont la pleine illustration : les introductions volontaires ou accidentelles ont profondément bouleversé les équilibres biologiques, causant des troubles et des dommages irréversibles, tel le lapin à Kerguelen durant la période de chasse à la baleine pour permettre la survie en cas de naufrage éventuel, mais aussi chèvres, moutons, mouflons, rennes et à Amsterdam, des bovins. Les équilibres se modifient, les sols s'érodent, la diversité biologique s'accroît dans une durée courte, des populations régressent (pétrels) à cause des rats puis des chats introduits pour chasser les rats, des insectes et des végétaux s'installent et prospèrent ${ }^{15}$. Les facteurs sont multiples: modifications climatiques (réchauffement sensible depuis le début des années 1970), les activités humaines liées à la recherche scientifique, l'élan touristique favorisé depuis 1994 par les rotations régulières du " bateau de souveraineté " ravitailleur (quatre rotations annuelles du Marion-Dufresne) et la visite de Crozet, Amsterdam et Kerguelen par des affrètements du tourisme de l'extrême. La vulnérabilité des écosystèmes, elle, s'amplifie.

13. Articles L. 331-1 et suivants exclus de la liste énumérative des dispositions applicables aux TAAF en vertu de l'article L. 640-1.

14. Sur cet insularity syndroma : $F$. Ramade, "Dictionnaire encyclopédique de l'écologie et des sciences de l'environnement ", Paris, Ediscience International, 1993, p. 660.

15. Cf. le site officiel des TAAF : WwN. Taaf.fr 
Le droit existant est complexe, peu lisible, révélant une certaine immaturité ${ }^{16}$. En vertu du principe de spécialité législative, à l'exception des lois de souveraineté, les lois nationales ne sont applicables, dans les TAAF, que par dispositions expresses ${ }^{17}$. Pour ce qui est du Code de l'environnement, il convient de se référer à l'article L. 640-1. Celui-ci fonde, par la technique du renvoi, les dispositions de ce code applicables de plein droit à ces territoires extrêmes : mécanismes de l'étude d'impact des travaux et projets d'aménagement (art. L. 122-1 à 3), régime juridique des associations de protection de l'environnement (art. L. 141-1 à 142-3), dispositions spéciales aux eaux marines et aux voies ouvertes à la navigation maritime (art. L. 218-1 à 72), stratégie de lutte contre l'effet de serre (art. L. 229-1 à 4), réserves naturelles nationale ou régionale (art. L. 332-1 à 27, excepté l'article relatif à l'obligation d'enfouissement des lignes électriques, ce qui se conçoit aisément), préservation du patrimoine biologique (art. L. 411-1 à 4), en excluant, malgré leur haut intérêt, les dispositions relatives aux inventaires scientifiques et celles à la surveillance biologique du territoire, enfin réglementation des activités humaines, des établissements détenant des animaux sauvages, de la conservation des habitats naturels, de la faune et de la flore, ainsi que le dispositif de répression pénale (art. L. 412-1 à L. 415-5) ${ }^{18}$.

De cette mosaïque législative, minimum permettant d'édifier un système de normes environnementales, l'on s'étonnera d'une curiosité, soit l'absence des principes directeurs du droit de l'environnement : précaution, prévention, pollueurpayeur et information et participation (art. L. 110-1). La Charte constitutionnelle de l'environnement, issue de la loi constitutionnelle $n^{\circ} 2005-205$ du $1^{\text {er }}$ mars $2005^{19}$, couvre cette carence du fait de son intégration au bloc de constitutionnalité.

En somme, les mesures existantes visent à établir un régime d'autorisation d'acces aux zones non fréquentées par les hivernants ${ }^{20}$, d'interdiction de la chasse et de l'introduction d'espèces non autochtones, de fixation de mesures de non-perturbation, telles les distances de sécurité pour approcher les animaux, d'interdiction de modifier les milieux par toute action néfaste, etc. Des listes d'espèces, technique classique du droit de l'environnement, créent la catégorie d'espèces protégées : arrêté du 27 juillet 1995 sur les mammifères marins (cétacés, éléphant de mer, léopard de mer...), arrêté du 14 août 1998 pour tous les oiseaux autochtones. La création de la réserve naturelle ne change pas profondément l'état du droit. Elle y met de la clarté et de la coherence, et le soumet à la tutelle du ministère de l'Écologie et du Développement durable. Nonobstant l'abrogation d'une aire de protection improprement dénommée, en droit, parc

16. D. Sombetzki-Lengagne, “ La protection de l'environnement dans les terres australes et antarctiques françaises : le projet de création d'une réserve naturelle des terres australes ", $R J E, n^{\circ} 3 / 2003$, p. 309 .

17. Ainsi du Code de l'action sociale et des familles (art. L. 581-1), du Code de la défense (art. L. 1671-1 et S., L. 2471-1 et S., L. 3571-1 et L. 5371-1 et S.), du Code de l'entrée et du séjour des étrangers et du droit d'asile (art. 765-1), du Code de l'organisation judiciaire (art. L. 935-1 et R. 935-1), du Code du patrimoine (art. L. 770-1 et S.), du Code de la recherche (art. L. 145-1, L. 355-1 et L. 445-1) et du Code de la santé publique (art. L. 1531-1 et S., L. 1535-1 et S., L. 1536-1, L. 2431-1 et S. L. 3831-1 et S. , L. 4431-1 et S., L. 5531-1).

18. Cet article L. 640-1 précise, en outre, que les pouvoirs dévolus normalement au préfet au titre des dispositions précitées, sont exercés par l'administrateur supérieur des TAAF. Par ailleurs, un sort particulier est fait à la protection des monuments naturels et sites en vertu de la loi n $56-1106$ du 3 novembre 1956.

19. JO du 2 mars.

20. Des zones à accès réglementé sont établies par l'arrêté nº 15 du 30 juillet 1985 (I'île aux Cochons à Crozet, les îles Nuageuses à Kerguelen...) et des zones sont réservées à la recherche scientifique par l'arrêté $n^{\circ} 14$ du 30 juillet 1985 (1'île Saint-Paul à Amsterdam, l'île Haute à Kerguelen...). 
national de refuge pour certaines espèces d'oiseaux et de mammifères ${ }^{21}$, la réserve établit un nouvel ordre juridique.

\section{B) STATUT ET RÉGIME JURIDIQUE DE LA RESSERVE NATURELLE DES TERRES AUSTRALES FRANÇAISES}

Cette réserve est la $156^{\circ}$ de France. C'est aujourd'hui la plus vaste avec 700000 hectares rien que pour la partie terrestre, la seule réserve ultra-marine inhabitée aussi. Elle s'inscrit dans le succes incontesté et justifié de l'instrument national de protection renforcée du patrimoine écologique. Son statut est simple, précis, pertinent et efficace. S'il existe des réserves naturelles régionales (art. L. 332-11 et S. du Code de l'environnement), anciennes réserves naturelles volontaires, l'essentiel de la mesure réside dans le statut général de réserve naturelle nationale. "Instrument spécifique de conservation des espèces et des milieux ${ }^{22}$, ce statut est élaboré en plusieurs phases : dérivée de la loi du 2 mai 1930 sur les sites et monuments naturels, accédant à l'autonomie comme espa-

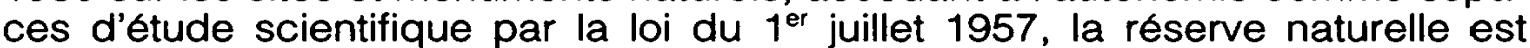
consacrée par les articles 16 et suivants de la grande loi $n^{\circ} 76-629$ du 10 juillet 1976 relative à la protection de la nature et devient un élément fondamental d'une politique nationale de conservation de l'environnement par la loi $n^{\circ} 2002-92$ du 22 janvier $2002^{23}$.

La réforme du statut des réserves naturelles par la loi du 27 février 2002 précitée prévoit qu'une telle décision de classement est prononcée par décret pour assurer la conservation d'éléments du milieu naturel d'intérêt national ou la mise en œuvre, notamment, d'une obligation résultant d'une convention internationale. Aussi, l'Etat français a-t-il opté pour l'institution d'une réserve naturelle nationale, en partie terrestre et en partie marine. Le champ d'application est immense : Saint-Paul et Amsterdam (eaux intérieures et mer territoriales incluses), archipel de Crozet (y compris les eaux territoriales sauf celles de l'île de la Possession), et Kerguelen (définition de la zone précise à l'art. $1^{\text {er }}$ dudit décret). L'Elat satisfait, par cette création, à ses obligations d'Etat cótier imposées par le droit international, l'obligation générale de protection de l'environnement en vertu de l'article 192 de la Convention des Nations Unies du 10 décembre 1982 sur le droit de la mer, et l'obligation de préserver la diversité biologique sur la base des articles 3 et 6 de la Convention de Rio du 22 mai 1992. Diverses recommandations l'y invitaient. Celle du Comité de l'environnement polaire en $1998^{24}$ ou encore celle de l'Union internationale de la conservation de la nature, organisation non gouvernementale d'autorité mondiale en la matière, aux termes de sa recommandation $n^{\circ} 01 / 11$ du 30 octobre $2001^{25}$; l'annonce officielle fut l'objet d'une intervention du président de la République lors de la Conférence internationale sur la biodiversité à Paris à I'UNESCO (janvier 2005).

21. Décret du 27 octobre 1938 abrogé par l'article 27 du décret du 3 octobre 2006. Si la terminologie ne désignait pas une catégorie juridique précise telle celle définie aux articles $L$. 331-1 et suivants du Code de l'environnement (inapplicables d'ailleurs dans les TAAF), il est indubitable que cette aire de protection est l'ancêtre de l'actuelle réserve naturelle nationale.

22. M. Prieur, « Droit de l'environnement $»$, Dalloz 2005, p. 443.

23. C. Cans, « Les réserves naturelles en 45 ans et deux mouvements : évolution, involution $\nsim, R J E$, $n^{\circ} 4 / 2002$, p. $551-588$.

24. Organe d'expertise crée par le décret no 93-740 du 29 mars 1993, mentionné dans les visas du decret de classement.

25. D. Sombetzki-Lengagne, * La protection de l'environnement dans les TAAF ^, op. cit., p. 311. 
Le principe de légalité est ici respecté, puisque l'article L. 332-1 du Code de l'environnement dispose que des parties du territoire d'une ou plusieurs communes peuvent faire l'objet d'un tel classement, y compris en affectant le domaine public et les eaux territoriales françaises. Si les motifs scientifiques sont incontestables et conformes à la loi, à la fois préservation du milieu naturel qui présente une importance particulière et volonté de le soustraire à toute intervention artificielle susceptible de le dégrader, l'on peut discuter de l'absence, et pour cause, d'au moins une commune. La formulation de l'article L. 332-1-I n'envisage pas les territoires inhabités, échappant à la territorialisation par une commune. Peut-être faul-il y voir d'ailleurs l'absence de mention, dans les listes récapitulatives des réserves naturelles existantes, des îles Eparses. Quoi qu'il en soit, si la lettre de la loi est ici source de vétilleuses spéculations, l'esprit de la loi doit primer. La préservation de l'environnement vaut une entorse à l'orthodoxie juridique. Et l'interprétation du juge administratif, compréhensive, irait à n'en pas douter dans ce sens ${ }^{26}$.

Quant à sa gestion, le décret de classement du 3 octobre 2006 adapte le régime juridique de la réserve à sa configuration naturelle. Outre la spécificité de son autorité compétente, l'administrateur supérieur des TAAF, le comité consultatif est le conseil consultatif des TAAF institué par la loi $n^{\circ}$ 55-1052 du 6 août 1955 précitée. Ce dernier donne son avis d'expert sur toute modalité de gestion de la réserve, en particulier sur le plan de gestion qui est l'outil décisif en la matière. Toutefois, sans qu'il soit fait mention d'une quelconque hiérarchie, cet avis est partagé avec le comité de l'environnement polaire institué par le décret $n^{\circ}$ 93-740 du 29 mars 1993 précité. Ce dernier, à la lecture combinée des articles 3 et 4 du décret de classement, paraît être l'autorité scientifique par excellence, l'essentiel restant que ces instances soient composées d'une part représentative de scientifiques qualifiés. Ces dispositions ne sont pas anodines. Le plan de gestion, en effet, doit être élaboré avant le 3 octobre 2009 par l'administrateur supérieur, sur la base d'une évaluation scientifique du patrimoine naturel et de son évolution; il fixe les objectifs de protection à atteindre. Ainsi, comme pour toute réserve naturelle nationale, elle devient ce que le plan de gestion prévoit. II doit être arrêté pour cinq ans par l'administrateur supérieur, après avis au Conseil national de la protection de la nature, et est révisé tous les cinq ans apres évaluation scientifique. Cette procédure est essentielle en ce qu'elle permet au droit d'être ce qu'il doit être, une fiction fondée sur les données de la science.

L'article L. 332-3 du Code de l'environnement détermine le régime juridique particulier auquel sont soumis les territoires classés. Les activités sont interdites ou réglementées. Le décret de classement du 3 octobre 2006 affine l'adaptation du droit à la configuration naturelle et aux nécessités écologiques puisqu'il distingue la partie terrestre de la partie marine. S'agissant de la première, les interdictions sont strictes, absolues, et justifiees (art. 6 et 7 ) : interdiction d'introduire des especes sauf missions de service public ou de sauvetage ou encore de ravitaillement, de porter atteinte aux espèces ou de les troubler (dérogations pour les plantes à des fins scientifiques, médicales ou paramédicales), interdiction de nuire à la qualité des écosystèmes (art. 10), interdiction de mener toute recher-

26. Contrôle normal sur l'intérêt scientifique justifiant le classement (CE, 14 novembre 1979, Cruse, RJE 1980, p. 185, note Charbonneau), jusqu'à la pertinence de la délimitation en application de la théorie du joyau et de l'écrin (CE, 29 juillet 1998, Mme Pesson, RJE 1999, p. 170, note Billet. Absence de motivation nécessaire, interprétatıon audacieuse des textes, $C E, 22$ janvier 2003. Commune de Vald'lsere et Association de montagnards de la Vanoise, JCP adm. 2003, $n^{\circ} 1561$, note Billet). 
che miniere et a fortiori toute exploitation (art. 11) et de collecter des minéraux et fossiles (sauf à des fins de recherche scientifique, art. 12), interdiction de toute activité industrielle et commerciale (sauf s'il y a compatibilité avec les objectifs de gestion, art. 13), interdiction enfin de la circulation des véhicules à moteur (sauf pour motifs d'entretien, de surveillance, de missions de service public, etc., art. 18 et 19). Le représentant de l'Etat doit, sur toutes ces dérogations, être très vigilant. Encore plus dans la détermination des activités non pas interdites mais réglementées en conformité avec le plan de gestion, et l'on notera la différence de degré entre compatibilité et conformité d'un point de vue de la contrariété des normes, telles que les activités agricoles, pastorales et aquacoles (art. 9), les activités de découverte du milieu et sportives (art. 14), les activités relatives à la médiatisation (art. 15 et 16), tout comme le stationnement et la circulation des personnes (art. 17). Les bases existantes, comme les futures, sont soumises à ce régime particulier nonobstant quelques particularités nécessaires à leur bon fonctionnement (art. 20), ainsi que la présence des forces armées sauf mission de défense ou de souveraineté (art. 26).

Tel est le régime juridique de la réserve naturelle sur sa partie terrestre, classique au demeurant, constitué d'interdictions plus ou moins absolues et de réglementations adaptées. Néanmoins, le décret de classement du 3 octobre 2006 crée une nouveaute. II institue des zones de protection intégrale (chapitre IV), où toute activité humaine est interdite, sauf dérogation accordée par l'administrateur supérieur sur demande motivée. En cas de force majeure ou de nécessité d'exercice de la souveraineté, cette restriction disparaît, en toute logique. Ainsi en est-il de l'intégralité de l'île de Saint-Paul et de parties de Crozet (île de l'Est, île des Apôtres...) et de Kerguelen (îles Nuageuses, île Cluny...). Ce double degré de protection, classique et plus ou moins relatif dans certaines zones, innovant et absolu dans d'autres, est remarquable. Si la base textuelle doit être recherchée dans les articles $L$. 332-16 à 18 et L. 332-3 combinés du Code de l'environnement, l'expression "zone de protection intégrale " est inexistante. L'on entend plus sûrement, et juridiquement, " périmètre de protection ".

\section{II. - LA PRÉSERVATION DES INTÉRÊTS ÉCONOMIQUES ACTUELS ET FUTURS DANS LES EAUX DES TERRES AUSTRALES FRANÇAISES COMBINEE AVEC LA PROTECTIÓN DE L'ENVIRONNEMENT}

La définition de la partie marine de la réserve naturelle est très limitée. N'appartiennent à la réserve marine (et encore pas toujours) que les eaux intérieures (en deçà des lignes de base) et les eaux territoriales (douze nautiques au-delà des lignes de base). Aucune allusion n'est faite aux zones économiques, ou parties des zones économiques (188 nautiques au-delà de la mer territoriale). De même, la réglementation de la partie marine de la réserve naturelle est également très succincte. Elle ne concerne que trois points: le mouillage des navires qui y est réglementé par le préfet de la Réunion ${ }^{27}$, les cétacés qui y bénéficient d'une protection absolue, la pêche qui peut y être réglementée ou interdite par

27. Rappelons qu'il est responsable de l'action de l'Etat en mer au titre de “ délégué du Gouvernement dans les eaux bordant les cótes des terres françaises de l'océan Indien et des Terres australes et antarctiques françaises ". C'est également le préfet de la Réunion qui dirige la zone de défense à laquelle appartiennent les TAAF. 
l'Administrateur supérieur des $\mathrm{TAAF}^{28}$. II n'échappe à personne, à la lecture du décret, que ce dernier ne vise en substance que les terres des îles considérées et qu'il ne vise que très marginalement les dépendances maritimes de ces mêmes terres. La réserve naturelle des terres australes françaises n'est donc pas une réserve maritime. Or il faut avoir à l'esprit que les îles australes sont d'une superficie terrestre très modeste $\left(7747 \mathrm{~km}^{2}\right)^{29}$ mais qu'elles génèrent des zones maritimes immenses ( $1861000 \mathrm{~km}^{2}$ de zones économiques).

\section{A) LES INTÉRÊTS ÉCONOMIQUES EN JEU}

Pour l'exploitation des fonds marins, les recherches à finalité économique en sont encore au stade expérimental, ou n'ont pas débouché sur une exploitation rentable dans un avenir proche. C'est le cas pour ce qui est des sites de nodules polymétalliques et pour la recherche d'hydrocarbures. Les ressources dans ce domaine de l'exploitation des fonds marins ne sont donc que potentielles. Néanmoins, les espoirs en la matière ne sont nullement abandonnés comme le prouvent les recherches entreprises au large des îles australes (principalement Kerguelen et Crozet) et visant à une prochaine demande d'extension de leur plateau continental au-delà des 200 nautiques ${ }^{30}$. II est clair qu'on ne demande pas une extension de plateau continental si on est décidé à renoncer à l'exploitation économique.

Pour l'exploitation des eaux surjacentes, par contre, la pêche est une activité économique bien réelle. Elle constitue le deuxieme poste d'exportation pour la Réunion, après la canne à sucre. Les deux postes principaux de cette activité concernent la langouste et la légine. La pêche à la langouste, pratiquée aussi bien dans les eaux territoriales que dans les zones économiques des îles SaintPaul et Amsterdam, est très ancienne. La campagne de pêche à la langouste dure cinq mois, de décembre à avril. La pêche à la légine, ou morue antarctique, est d'apparition relativement récente ${ }^{31}$. La légine (Dissostichus eleginoides) est un poisson à chair grasse encore peu connu en Europe, mais déjà très recherché sur les marchés asiatiques. Son exploitation est particulièrement rentable. Pour la campagne de pêche 2005-2006, le total admissible de capture de légine dans les zones économiques de Kerguelen et de Crozet était fixé à 6150 tonnes par l'Administrateur supérieur. II est fixé à 6100 tonnes pour 2006-2007 ${ }^{32}$. A titre de comparaison, il était de 6200 tonnes en 1997-1998. Le prélèvement légal reste donc sensiblement le même depuis une dizaine d'années. En réalité, le prélèvement global a été divisé par cing ou six du fait de la disparition quasi-totale de la pêche illicite ${ }^{33}$ grâce au renforcement du dispositif pénal français, à la coopération internationale dans la zone et surtout grâce à l'action énergique de

28. Le principe est donc l'autorisation de la pêche, l'exception est la réglementation de la pêche ou son interdiction. En fait et depuis des décennies, la pêche de poissons et de langoustes est autorisée dans les eaux de Saint-Paul et Amsterdam, elle est interdite dans les eaux de Crozet et Kerguelen.

29. A titre de comparaison, la Corse a une superficie de $8750 \mathrm{~km}^{2}$.

30. La France vise une extension de son plateau continental au large de ses îles australes de Crozet et Kerguelen (campagnes Kergueplac 1 et 2 du programme Extraplac). Une telle extension peut atteindre théoriquement jusqu'à 150 nautiques au-delà de la ZEE. Les demandes doivent étre déposées avant 2009 devant la Commission du Plateau continental instituee par la Convention de Montego Bay.

31. C'est en 1990 qu'un bateau de la société de pêche SAPMER découvre l'existence de légine sur le plateau continental des îles Kerguelen et Crozet.

32. Auxquelles s'ajouteront exceptionnellement 300 tonnes au titre de l'évaluation scientifique de la biomasse, arrêté de l'Administrateur supérieur 2006-39 du 30 août 2006.

33. Cf. M. Manouvel, "Le territoire d'outre-mer des Terres australes et antarctiques françaises $», o p$. cit., p. 169-189. 
la marine nationale ${ }^{34}$. La gestion de la ressource semble donc être prudente, cette prudence étant due à l'observation des conclusions des recherches scientifiques tant nationales qu'internationales sur la question.

\section{B) LA PRISE EN COMPTE NATIONALE ET INTERNATIONALE DE LA PRÉSERVATION DE L'ENVIRONNEMENT}

La pêche dans les eaux des TAAF est réglementée de manière précise. Cette réglementation est édictée par une autorité nationale, l'Administrateur supérieur, mais elle répond néanmoins à des obligations souscrites par la France dans le cadre d'accords internationaux. II convient, en premier lieu, de faire référence à la Convention sur le droit de la mer, qui prévoit : « L'Etat côtier, compte tenu des données scientifiques les plus fiables dont il dispose, prend des mesures appropriées de conservation et de gestion pour éviter que le maintien des ressources biologiques de sa zone économique exclusive ne soit compromis par une surexploitation. L'Etat côtier et les organisations internationales compétentes, sous-régionales, régionales ou mondiales, coopèrent selon qu'il convient à cette fin ${ }^{35}$.

Il est nécessaire, en second lieu, de faire référence à la Convention sur la conservation de la faune et de la flore marines de l'Antarctique ${ }^{36}$, signée à Canberra le 20 mai 1980 par quinze Etats, dont la France, et qui est entree en vigueur le 7 avril $1982^{37}$. La Convention, comme son nom l'indique, a pour but de préserver la vie marine dans les zones considérées. II s'agit principalement de faire en sorte que l'exploitation économique des ressources marines ne mette pas en cause la pérennité de ces dernières. L'exploitation économique n'est donc pas interdite mais elle est fortement réglementée. Cette Convention met en place, pour la réalisation de son objectif, une Commission de conservation, chargée principalement d'études scientifiques, mais ayant aussi le pouvoir d'arrêter des mesures de conservation et d'organiser un système d'inspection ${ }^{38}$. Cette Commission pour la conservation de la faune et de la flore marines de l'Antarctique, organe central du système nouveau, a son siège à Hobart, dans lîle australienne de Tasmanie ${ }^{39}$. Elle est assistée par un Comité scientifique et par un Secrétariat.

Si la Convention fait partie du système antarctique, elle intéresse également une zone dépassant le cadre géographique habituel de ce système ${ }^{40}$. En effet, les îles des TAAF concernées par la Convention, Kerguelen et Crozet, sont, comme nous l'avons mentionné, situées bien au nord du $60^{\mathrm{e}}$ parallèle Sud. II est vrai que les deux archipels sont soumis à un régime dérogatoire, puisqu'en vertu d'une

34. De plus, un traité franco-australien de coopération impliquant des actions conjuguées des deux marines a été signé en 2003 (les zones économiques des îles australes Heard et MacDonald - possessions australiennes - étaient également victimes de la pêche illicite). Il est entré en vigueur en janvier 2005.

35. Article 61, sur la conservation des ressources biologiques, dans son alinéa 2

36. Convention on the Conservation of Antarctic Marine Living Resources.

37. Etaient invitées à Canberra les treize parties consultatives du Traité de Washington et les deux Allemagne qui allaient le devenir. En 2006, la Convention réunit 34 Etats parties.

38. C'est ainsi que pour 2005-2006, pas moins de 64 mesures de conservation régissaient les activités dans la zone couverte par la Convention.

39. La dénomination courante française de cette commission est "Camélar ", phonétiquement proche du sigle anglais " CCAMLR " (Commission for the Conservation of Antarctic Marine Living Resources).

40. Nous sommes ici dans la zone de convergence antarctique (front polaire). rencontre des eaux froides de l'océan Austral et des eaux moins froides du Nord. C'est cette zone de convergence, véritable barriere biologique, qui fait de l'océan Austral un écosystème fermé. 
déclaration du président de la Conférence préparatoire, faite le 19 mai 1980 et intégrée dans l'acte final, l'application de mesures aux archipels de Kerguelen et Crozet nécessite l'approbation de la France. Cette dernière reste donc libre de sa réglementation à moins qu'elle ne soit contraire à des décisions qu'elle a précédemment approuvées. Ainsi, en 1990, la France s'oppose à des mesures proposées par la Commission concernant les stocks de poissons autour des îles de l'archipel des Kerguelen. Depuis lors, les mesures de conservation appliquées aux sous-zones statistiques comprenant Kerguelen et Crozet (sous-zone statistique 58.5.1 pour Kerguelen et sous-zone statistique 58.6 pour Crozet) précisent que les mesures édictées ne s'étendent pas aux eaux adjacentes aux deux îles. C'est le cas notamment de la mesure de conservation qui interdit la pêche à la légine dans les deux sous-zones statistiques auxquelles appartiennent Crozet et Kerguelen.

C'est dans l'esprit de ces Conventions internationales, qui font primer le souci de conservation et de protection du milieu marin sur celui de son exploitation, que le décret du 27 mars 1996, relatif aux conditions d'exercice de la pêche maritime dans les TAAF, prévoyait dans son article 2 : « L'Administrateur supérieur des Terres australes et antarctiques françaises délivre les autorisations de pêche sous forme de licence ou de permis et détermine les règles et les interdictions relatives à l'exercice de la pêche maritime, par arrêté pris après avis du Muséum national d'histoire naturelle et avec l'accord du ministre chargé de la pêche, du ministre chargé de l'outre-mer et du ministre des affaires étrangères " ${ }^{41}$. II revient donc au responsable du Laboratoire d'ichtyologie générale et appliquée du Muséum national d'histoire naturelle de Paris, actuellement le professeur Guy Duhamel, de donner un avis sur la fixation des zones de pêche autorisée, sur les modalités de la pêche, ainsi que sur les quotas annuels de prise à l'intérieur de ces zones, et ce dans le souci d'assurer la pérennité de la ressource. L'avis du Muséum national d'histoire naturelle n'est certes qu'un avis consultatif, mais il est, en fait, toujours suivi. C'est dans ce contexte que s'inscrit la réserve naturelle des Terres australes.

Les tentatives répétées pour promouvoir des activités économiques sur les îles australes elles-mêmes s'étant révélées vaines, l'instauration d'une réserve naturelle sur le territoire terrestre de ces îles en était donc facilitée et ce d'autant plus que toutes les terres en question appartiennent au domaine public. L'habituel antagonisme économie-écologie n'avait donc pas lieu d'être. II n'en allait pas de même, par contre, pour ce qui est des zones maritimes entourant ces îles australes.

Au nom du développement durable, objectif que doivent promouvoir les pouvoirs publics en vertu de l'article 5 de la charte constitutionnelle du $1^{\text {er }}$ mars 2005 précitée, la réserve naturelle des Terres australes détermine des degrés dans la protection, adaptés à la situation : intégrale ici, réglementée là. Un nouvel ordre juridique s'établit en conciliant les exigences de la préservation de la biodiversité et les potentialités d'exploitation des ressources halieutiques. L'important est la structure de la réponse apportée : la réserve naturelle crée un statut juridique du milieu en lui attribuant une valeur d'universalité patrimoniale ${ }^{42}$, indice de la modernité du droit de l'environnement.

41. JO du 29 mars 1966, p. 1800. Il est intéressant de noter que le premier visa du décret renvoie à la Convention de Canberra du 20 mai 1980.

42. F. Ost, * La nature hors la loi. L'écologie à l'épreuve du droit », Paris, La Découverte, 1995, p. 320. 